\title{
EXPLICIT KUMMER THEORY FOR THE RATIONAL NUMBERS
}

\author{
ANTONELLA PERUCCA, PIETRO SGOBBA, SEBASTIANO TRONTO
}

\begin{abstract}
Let $G$ be a finitely generated multiplicative subgroup of $\mathbb{Q}^{\times}$having rank $r$. The ratio between $n^{r}$ and the Kummer degree $\left[\mathbb{Q}\left(\zeta_{m}, \sqrt[n]{G}\right): \mathbb{Q}\left(\zeta_{m}\right)\right]$, where $n$ divides $m$, is bounded independently of $n$ and $m$. We prove that there exist integers $m_{0}, n_{0}$ such that the above ratio depends only on $G, \operatorname{gcd}\left(m, m_{0}\right)$, and $\operatorname{gcd}\left(n, n_{0}\right)$. Our results are very explicit and they yield an algorithm that provides formulas for all the above Kummer degrees (the formulas involve a finite case distinction).
\end{abstract}

\section{INTRODUCTION}

The aim of this paper is developing a theory that allows to explicitly compute the degree of Kummer extensions. Let $G$ be a finitely generated multiplicative subgroup of $\mathbb{Q}^{\times}$having positive rank $r$ and, without loss of generality, not containing -1 . We are interested in the Kummer extension

$$
\left[\mathbb{Q}\left(\zeta_{M}, \sqrt[N]{G}\right): \mathbb{Q}\left(\zeta_{M}\right)\right] \quad \text { with } N \mid M
$$

where $\zeta_{M}$ is a root of unity of order $M$, and where we are adding the $N$-th roots of all elements of $G$. The maximal possible value for the Kummer degree (1.1) is $N^{r}$, and in general this degree is a divisor of $N^{r}$. It is known (see [4, Theorem 3.1] for a direct proof) that the ratio between $N^{r}$ and the Kummer degree (1.1) is bounded independently of $N$ and $M$. We interpret this ratio as the failure of maximality for the Kummer degree.

We prove that there exist explicitly computable integers $M_{0}, N_{0}$ such that the failure of maximality for the Kummer degree only depends on $M$ and $N$ through $\operatorname{gcd}\left(M, M_{0}\right)$ and $\operatorname{gcd}\left(N, N_{0}\right)$. We also present a strategy to provide formulas for the Kummer degree in (1.1): the input is the group $G$, and the output are formulas for all $M, N$ with a finite case distinction. This algorithm has been implemented in Sagemath by Tronto. Notice that the computation of one single degree (i.e. fixing the parameters $M, N$ and with $M=N$ ) has also been obtained by Palenstijn in his thesis [2] with a different method (namely with the theory of entanglement groups due to Lenstra).

Let us now illustrate why the Kummer degree fails to be maximal. We may suppose without loss of generality that $N:=\ell^{e}$ for some prime number $\ell$. We distinguish two reasons for the failure of maximality for the Kummer degree, namely the $\ell$-adic failure and the adelic failure. The $\ell$-adic failure is due to divisibility properties involving the number $\ell$. For example, if

2010 Mathematics Subject Classification. Primary: 11Y40; Secondary: 11R18, 11R21.

Key words and phrases. Number fields, Kummer theory, Degree, Cyclotomic fields. 
$G=\left\langle 5^{\ell}\right\rangle$, then for all $e \geqslant 1$ we have

$$
\left[\mathbb{Q}\left(\zeta_{\ell^{e}}, \sqrt[\ell^{e}]{5^{\ell}}\right): \mathbb{Q}\left(\zeta_{\ell^{e}}\right)\right]=\ell^{e-1}
$$

The adelic failure is due to the fact that the square root of any rational number is contained in some cyclotomic field. For example, if $G=\langle 5\rangle$, we need to take into account that $\sqrt{5}$ lies in $\mathbb{Q}\left(\zeta_{5}\right)$ :

$$
\left[\mathbb{Q}\left(\zeta_{M}, \sqrt[N]{5}\right): \mathbb{Q}\left(\zeta_{M}\right)\right]= \begin{cases}N, & \text { if } N \text { is odd or } 5 \nmid M \\ N / 2, & \text { otherwise. }\end{cases}
$$

The structure of the paper is as follows. In Section 2 we introduce the notation that is used in the rest of the paper, and in particular we write $A_{\ell}(N)$ for the $\ell$-adic failure, and $B(M, N)$ for the adelic failure, and we consider the Kummer failure $C(M, N)$ (see also Theorem 2.1 and (2.2), which is

$$
C(M, N):=\frac{N^{r}}{\left[\mathbb{Q}\left(\zeta_{M}, \sqrt[N]{G}\right): \mathbb{Q}\left(\zeta_{M}\right)\right]}=B(M, N) \cdot \prod_{\ell \mid N} A_{\ell}(N) .
$$

Notice that knowing $C(M, N)$ for every $M, N$ with $N \mid M$ is equivalent to knowing the Kummer degrees that we are interested in. Also notice that the assumption that $G$ is torsionfree is not really necessary (see Remark 2.5).

Section 3 is devoted to studying the $\ell$-adic failure $A_{\ell}(N)$ for all odd prime numbers $\ell$ (notice that it equals 1 for all but finitely many primes $\ell$ ). The 2 -adic failure $A_{2}(N)$ is studied in Section 4. The adelic failure $B(M, N)$ is more complicated and is studied in the following two sections. Then in Section 7 we prove in particular the following result:

Theorem 1.1. There are integers $M_{0}$ and $N_{0}$, depending only on $G$, such that for all integers $N, M$ with $N \mid M$, the Kummer failure $C(M, N)$ depends only on $\operatorname{gcd}\left(M, M_{0}\right)$ and on $\operatorname{gcd}\left(N, N_{0}\right)$.

Finally, the last section is devoted to examples that give an insight on the case distinction in our results.

\section{THE FAILURE OF MAXIMALITY FOR KUMMER EXTENSIONS}

We make use of the following standard notation for any positive integers $N, M$ and any prime number $\ell$ : we denote by $\zeta_{M}$ a primitive $M$-th root of unity; $v_{\ell}(N)$ is the $\ell$-adic valuation of $N ;(N, M)$ or $\operatorname{gcd}(N, M)$ is the greatest common divisor of $N$ and $M$, while $[N, M]$ or $\operatorname{lcm}(M, N)$ is the least common multiple; for $n \geqslant 1$ we write $\ell^{n} \| N$ to mean $v_{\ell}(N)=n$.

Let $G$ be a finitely generated and torsion-free subgroup of $\mathbb{Q}^{\times}$of positive rank $r$. We denote the $M$-th cyclotomic field by $\mathbb{Q}_{M}:=\mathbb{Q}\left(\zeta_{M}\right)$ and, for $N \mid M$, the $N$-th Kummer extension of $\mathbb{Q}_{M}$ related to $G$ by $\mathbb{Q}_{M, N}:=\mathbb{Q}_{M}(\sqrt[N]{G})$.

Theorem 2.1 (see [4, Theorem 3.1] for a direct proof). Let $G$ be a finitely generated and torsion-free subgroup of $\mathbb{Q}^{\times}$of positive rank $r$. For all integers $M, N \geqslant 1$ with $N \mid M$ the 
Kummer failure

is bounded independently of $M, N$.

$$
C(M, N):=\frac{N^{r}}{\left[\mathbb{Q}_{M, N}: \mathbb{Q}_{M}\right]}
$$

Using arguments of elementary field theory and considering the prime factorization $N=$ $\prod_{\ell} \ell^{n}$, where $n=v_{\ell}(N)$, we can write

$$
C(M, N)=\prod_{\ell \mid N} \frac{\ell^{n r}}{\left[\mathbb{Q}_{M, \ell^{n}}: \mathbb{Q}_{M}\right]}=\prod_{\ell \mid N} \frac{\ell^{n r}}{\left[\mathbb{Q}_{\ell^{n}, \ell^{n}}: \mathbb{Q}_{\ell^{n}}\right]} \cdot \frac{\left[\mathbb{Q}_{\ell^{n}, \ell^{n}}: \mathbb{Q}_{\ell^{n}}\right]}{\left[\mathbb{Q}_{M, \ell^{n}}: \mathbb{Q}_{M}\right]} .
$$

We then decompose the Kummer failure:

Definition 2.2. Let $\ell$ be a prime number. Let $N \geqslant 1$ with $v_{\ell}(N)=n$. The $\ell$-adic failure $A_{\ell}(N)$ at $N$ is defined as

$$
A_{\ell}(N):=C\left(\ell^{n}, \ell^{n}\right)=\frac{\ell^{n r}}{\left[\mathbb{Q}_{\ell^{n}, \ell^{n}}: \mathbb{Q}_{\ell^{n}}\right]} .
$$

Notice that the integer $A_{\ell}(N)$ is a power of $\ell$ that depends on $N$ only through its $\ell$-adic valuation.

Definition 2.3. Let $N \geqslant 1$ with $v_{2}(N)=n$, and let $M \geqslant 1$ with $N \mid M$. The adelic failure at $M, N$ is defined as the ratio

$$
B(M, N):=\frac{\left[\mathbb{Q}_{2^{n}, 2^{n}}: \mathbb{Q}_{2^{n}}\right]}{\left[\mathbb{Q}_{M, 2^{n}}: \mathbb{Q}_{M}\right]}
$$

Notice that the integer $B(M, N)$ is a power of 2 , and that we have

$$
B(M, N)=\left[\mathbb{Q}_{2^{n}, 2^{n}} \cap \mathbb{Q}_{M}: \mathbb{Q}_{2^{n}}\right] .
$$

The ratio $\left[\mathbb{Q}_{\ell^{n}, \ell^{n}}: \mathbb{Q}_{\ell^{n}}\right] /\left[\mathbb{Q}_{M, \ell^{n}}: \mathbb{Q}_{M}\right]$ equals 1 if $\ell$ is odd by [4, Lemma 3.5], so that we have

$$
C(M, N)=B(M, N) \cdot \prod_{\ell \mid N} A_{\ell}(N) .
$$

Lemma 2.4 (cf. [4, Lemmas 3.2, 3.5]). Let $\ell$ be a prime number.

(1) The $\ell$-adic failure $A_{\ell}(N)$ is bounded independently of $N$. More precisely, there is an integer $n_{\ell} \geqslant 0$ (which depends only on $G$ and $\ell$ ) such that for every $N \geqslant 1$ we have $A_{\ell}(N) \mid \ell^{n_{\ell}}$. The integer $n_{\ell}$ equals 0 for all but finitely many primes $\ell$.

(2) The adelic failure $B(M, N)$ is bounded independently of $M, N$. More precisely, for all $M, N \geqslant 1$ with $N \mid M$, we have $B(M, N) \mid 2^{r}$, where $r$ is the rank of $G$.

Notice that by Corollary 7.3 we have the following stronger statements:

(1) Let $\ell$ be a prime number. There is an integer $\alpha_{\ell}$ such that $A_{\ell}(N)=\alpha_{\ell}$ for all $N$ with $v_{\ell}(N) \geqslant c_{\ell}$, where $c_{\ell}$ is some integer depending only on $\ell$ and $G$. We call $\alpha_{\ell}$ the total $\ell$-adic failure. The integer $\alpha_{\ell}$ is a power of $\ell$, and we have $\alpha_{\ell}=1$ for all but finitely many primes $\ell$. 
(2) There is an integer $\beta$ such that $B(M, N)=\beta$ for all $M, N$ with $N\left|M, M_{0}\right| M$ and $N_{0} \mid N$, where $M_{0}$ and $N_{0}$ are some integers depending only on $G$. We call $\beta$ the total adelic failure. In particular, $\beta$ is a power of 2 and we have

$$
\beta=\left[\mathbb{Q}_{2^{n}, 2^{n}} \cap \mathbb{Q}_{\infty}: \mathbb{Q}_{2^{n}}\right]
$$

for all sufficiently large $n$, where $\mathbb{Q}_{\infty}$ is the compositum of all cyclotomic fields.

Notice that $A_{\ell}\left(\ell^{n}\right)$ is non-decreasing in $n$, and in particular we have $\alpha_{\ell}=\max _{N} A_{\ell}(N)$. Conversely, in general $\beta$ cannot be expressed as the maximum value of $B(M, N)$ over $M, N$. For example, taking $G=\langle 2\rangle$ and $M=N=2^{n}$, the right-hand side of (2.1) is 2 if $n=1,2$, and it is 1 for $n \geqslant 3\left(\sqrt{2} \in \mathbb{Q}_{8}\right.$ gives rise to a 2 -adic failure instead).

In view of (2.2) we also define the total Kummer failure for $G$ by

$$
C_{0}:=\beta \cdot \prod_{\ell} \alpha_{\ell}
$$

where the product runs over all prime numbers $\ell$. In fact, the integer $C_{0}$ is such that $C(M, N)=$ $C_{0}$ for all $M, N$ with $N\left|M, M_{0}\right| M$ and $N_{0} \mid N$, where $M_{0}$ and $N_{0}$ depend only on $G$.

Remark 2.5. To deal with a finitely generated subgroup $G^{\prime}$ of $\mathbb{Q}^{\times}$which is not torsion-free, write $G^{\prime}=\langle-1\rangle \times G$, where $G$ is torsion-free. For $M, N$ with $N \mid M$ we have

$$
\left[\mathbb{Q}_{M}\left(\sqrt[N]{G^{\prime}}\right): \mathbb{Q}_{M}\right]=\left[\mathbb{Q}_{[M, 2 N]}(\sqrt[N]{G}): \mathbb{Q}_{[M, 2 N]}\right] \cdot\left[\mathbb{Q}_{[M, 2 N]}: \mathbb{Q}_{M}\right] .
$$

Therefore, the Kummer degree for $G^{\prime}$ is the product of a Kummer degree for the torsion-free group $G$ times $\varphi([M, 2 N]) / \varphi(M) \in\{1,2\}$.

\section{THE $\ell$-ADIC FAILURE FOR $\ell$ ODD}

Let $G$ be a finitely generated and torsion-free subgroup of $\mathbb{Q}^{\times}$of rank $r$ and let $\ell$ be an odd prime number. In order to compute the $\ell$-adic failure $A_{\ell}(N)$, we show how to determine the degrees

$$
\left[\mathbb{Q}_{\ell^{m}}\left(\sqrt[\ell^{n}]{G}\right): \mathbb{Q}_{\ell^{m}}\right]
$$

for all integers $m \geqslant n \geqslant 1$ using the results from [1, Section 3.3] which we recall in Proposition 3.1 .

An element of $\mathbb{Q}^{\times}$is called strongly $\ell$-indivisible if it is not an $\ell$-th power in $\mathbb{Q}^{\times}$. We call $a_{1}, \ldots, a_{r} \in \mathbb{Q}^{\times}$strongly $\ell$-independent if $a_{1}^{e_{1}} \cdots a_{r}^{e_{r}}$ is strongly $\ell$-indivisible whenever the integers $e_{1}, \ldots, e_{r}$ are not all divisible by $\ell$. We now specialize results of [1] for the rational numbers.

There is a basis $g_{1}, \ldots, g_{r}$ of $G$, which we call an $\ell$-good basis, such that

$$
g_{i}=b_{i}^{\ell^{d_{i}}}
$$

holds for some strongly $\ell$-independent elements $b_{1}, \ldots, b_{r}$ of $\mathbb{Q}^{\times}$, and for some non-negative integers $d_{i}$. We refer to the tuple $\left(d_{1}, \ldots, d_{r}\right)$ as the $d$-parameters for the $\ell$-divisibility of $G$. $\mathrm{Up}$ to reordering the elements of the basis, we choose the $d$-parameters to be a non-decreasing sequence: then they are the same for every $\ell$-good basis of $G$. 
Proposition 3.1 (cf. [1, Example 21]). Let $G$ be a finitely generated and torsion-free subgroup of $\mathbb{Q}^{\times}$. Let $\ell$ be an odd prime, and let $d_{1}, \ldots, d_{r}$ be the d-parameters for the $\ell$-divisibility of $G$. Then we have

$$
v_{\ell}\left(\left[\mathbb{Q}_{\ell^{m}, \ell^{n}}: \mathbb{Q}_{\ell^{m}}\right]\right)=\sum_{i=1}^{r} \max \left(n-d_{i}, 0\right) .
$$

In particular, the total $\ell$-adic failure is $\alpha_{\ell}=\ell^{\sum_{i} d_{i}}$.

Since the $d$-parameters for the $\ell$-divisibility of $G$ are explicitly computable (cf. [1, Section 6.1]), so is the $\ell$-adic failure for each odd prime $\ell$. The challenge is here computing the $\ell$-adic failure for all primes $\ell$ at once with a finite procedure. We consider a basis $g_{1}, \ldots, g_{r}$ of $G$, and we write the prime factorization of each generator $g_{i}$ as

$$
g_{i}= \pm \prod_{j=1}^{s} p_{j}^{e_{i j}}
$$

for some integers $e_{i j} \in \mathbb{Z}$, where $p_{j}$ runs through the finitely many primes appearing in the factorizations of the $g_{i}$ 's. We call $\left(e_{i j}\right)$, which is an $r \times s$ matrix with $r \leqslant s$, the matrix of the exponents.

Lemma 3.2. Let $\ell$ be an odd prime number and let $\left(e_{i j}\right)$ be the matrix of the exponents of a basis of $G$. If that matrix modulo $\ell$ has maximal rank, then the basis is an $\ell$-good basis of $G$ and the d-parameters for the $\ell$-divisibility are all zero.

Proof. The matrix of the exponents has maximal rank $r$ over $\mathbb{Z} / \ell \mathbb{Z}$ if and only if the vectors $v_{i}=\left(e_{i, 1}, \ldots, e_{i, s}\right)$ are linearly independent over $\mathbb{Z} / \ell \mathbb{Z}$. This means that if $\sum_{i} x_{i} v_{i} \equiv 0 \bmod$ $\ell$ for some integers $x_{i}$, then $\ell \mid x_{i}$ for every $i$. So the linear independence of the vectors $v_{i}$ is equivalent to the following condition: if there are some integers $x_{i}$ such that

$$
g:=\prod_{i} g_{i}^{x_{i}}= \pm \prod_{j} p_{j}^{\sum_{i} x_{i} e_{i j}}
$$

with $\ell \mid \sum_{i} x_{i} e_{i j}$ for all $j$, so that $g$ is an $\ell$-th power in $\mathbb{Q}$, then $\ell \mid x_{i}$ for all $i$. By definition, this means that the $g_{i}$ 's are strongly $\ell$-independent, which implies that the $d$-parameters are all zero.

The matrix of the exponents modulo $\ell$ has maximal rank $r$ for all but finitely many odd primes $\ell$, and the set of exceptions is easily computable. In particular, for all but finitely many primes $\ell$ the $d$-parameters are all zero, which gives

$$
\left[\mathbb{Q}_{\ell^{m}, \ell^{n}}: \mathbb{Q}_{\ell^{m}}\right]=\ell^{n} \quad \text { for all } m \geqslant n \geqslant 1
$$

by Proposition 3.1. Now consider one of the remaining odd primes $\ell$. We can apply the algorithm described in [1, Section 6.1] to compute an $\ell$-good basis for $G$ and the $d$-parameters for the $\ell$-divisibility. Applying Proposition 3.1 we can then compute $A_{\ell}(N)$ for every $N$. 


\section{THE 2-ADIC FAILURE}

Let $G$ be a finitely generated and torsion-free subgroup of $\mathbb{Q}^{\times}$of rank $r$. In this section we show how to compute the degrees

$$
\left[\mathbb{Q}_{2^{m}}\left(\sqrt[2^{n}]{G}\right): \mathbb{Q}_{2^{m}}\right]
$$

for all integers $m \geqslant n \geqslant 1$. In Theorem 4.2 we recall from [1, Section 3.3] parametric formulas for these degrees: for $m \geqslant 2$ we can work over $\mathbb{Q}_{4}$ and apply the formula (4.2), while for $m=n=1$, we can make use of Eq. 4.3.

Let $K$ be either $\mathbb{Q}^{\times}$or $\mathbb{Q}_{4}^{\times}$. An element of $K$ is called strongly 2-indivisible in $K$ if it is not a square in $K^{\times}$times a root of unity in $K$. Elements of $K^{\times}$are strongly 2-independent if the product of any nonempty subset of them is strongly 2 -indivisible. We recall results of [1] for $K$.

We consider a finitely generated and torsion-free subgroup $G$ of $K^{\times}$and a basis $g_{1}, \ldots, g_{r}$ of $G$. We can write

$$
g_{i}=\zeta_{2^{h_{i}}} \cdot b_{i}^{2^{d_{i}}}
$$

for some strongly 2-indivisible elements $b_{1}, \ldots, b_{r}$ of $K^{\times}$, for some non-negative integers $d_{i}$ and for some roots of unity $\zeta_{2^{h_{i}}}$ in $K$ of order $2^{h_{i}}$. We refer to $b_{i}$ as the strongly 2-indivisible part of $g_{i}$.

(1) We call $g_{1}, \ldots, g_{r}$ a 2-good basis of $G$ if the $b_{i}$ 's are strongly 2-independent. Recall from [1, Theorem 14] that a 2-good basis of $G$ always exists.

(2) For a 2-good basis of $G$, we refer to the tuple $\left(d_{1}, \ldots, d_{r} ; h_{1}, \ldots, h_{r}\right)$ as the parameters for the 2-divisibility of $G$ in $K$. We call $d_{1}, \ldots, d_{r}$ the $d$-parameters and $h_{1}, \ldots, h_{r}$ the $h$-parameters. Up to reordering the elements of the basis, we choose the $d$-parameters to be a non-decreasing sequence. Then the $d$-parameters are the same for every 2-good basis.

(3) Given a basis of $G$ written as in (4.1), the following are equivalent:

(a) The strongly 2 -indivisible parts $b_{1}, \ldots, b_{r}$ are strongly 2 -independent.

(b) The sum $\sum_{i} d_{i}$ is maximal (among all possible bases for $G$ ).

(c) The strongly 2 -indivisible parts $b_{1}, \ldots, b_{r}$ generate a torsion-free subgroup of $K^{\times}$ of rank $r$ and whose $d$-divisibility parameters are all zero.

Notice that the parameters for the 2-divisibility of $G$ are explicitly computable by the algorithm described in [1, Section 6.1].

Remark 4.1. Let $G$ be a finitely generated and torsion-free subgroup of $\mathbb{Q}^{\times}$, and suppose that $G$ contains negative elements. Then there is a 2-good basis of $G$ such that exactly one of the generators is negative. Indeed, consider any 2-good basis. If $g$ is one of the negative generators with the highest d-parameter, then we can simply multiply all other negative generators by $g$ (notice that we do not decrease their 2-divisibility).

Theorem 4.2 ([1, Theorem 18, Lemma 19]). Let $G$ be a finitely generated and torsion-free subgroup of $\mathbb{Q}^{\times}$of rank $r$. Let $m \geqslant n$ be positive integers. 
- If $m \geqslant 2$, we have

$v_{2}\left(\left[\mathbb{Q}_{2^{m}}\left(\sqrt[2^{n}]{G}\right): \mathbb{Q}_{2^{m}}\right]\right)=\max \left\{h_{i}+n_{i}: 1 \leqslant i \leqslant r\right\} \cup\{m\}-m+r n-\sum_{i=1}^{r} n_{i}$

where $n_{i}=\min \left(n, d_{i}\right)$ and $\left(d_{1}, \ldots, d_{r} ; h_{1}, \ldots, h_{r}\right)$ are the parameters for the 2 divisibility of $G$ in $\mathbb{Q}_{4}$.

- If $m=n=1$, we have

$$
[\mathbb{Q}(\sqrt{G}): \mathbb{Q}]=e\left[\mathbb{Q}_{4}(\sqrt{G}): \mathbb{Q}_{4}\right],
$$

where $e=2$ if $G$ contains minus a square in $\mathbb{Q}^{\times}$and $e=1$ otherwise.

Notice that to compute the divisibility parameters of $G$ over $\mathbb{Q}_{4}$ one only needs to take into account that, up to squares in $\mathbb{Q}^{\times}$, only the elements \pm 2 are strongly 2 -indivisible over $\mathbb{Q}$ but not over $\mathbb{Q}_{4}$. Nevertheless, in [5] we show in a more general setting that one can compute the degrees in (4.2) using the parameters over $\mathbb{Q}$ and certain properties of $G$.

\section{THE INTERSECTION BETWEEN KUMMER EXTENSIONS AND CYCLOTOMIC FIELDS}

This section is devoted to studying the intersection

$$
\mathbb{Q}_{2^{m}}\left(\sqrt[2^{n}]{G}\right) \cap \mathbb{Q}_{\infty}
$$

where $n \leqslant m$ are positive integers, and where $\mathbb{Q}_{\infty}$ denotes the compositum of all cyclotomic fields.

Notation. If $\left\{g_{i}\right\}$ is a basis of $G$, then we write $g_{i}=g_{i, d_{i}}$ to display the $d$-parameter for the 2-divisibility of $g_{i}$ in $\mathbb{Q}$.

Firstly we deal with the case $G \subseteq \mathbb{Q}_{+}^{\times}$.

Theorem 5.1. Let $G$ be a finitely generated and torsion-free subgroup of $\mathbb{Q}^{\times}$of positive rank. Suppose that $G$ contains only positive elements. Then for every 2-good basis $\left\{g_{i, d_{i}}\right\}$ we have

$$
\mathbb{Q}_{2^{m}, 2^{n}} \cap \mathbb{Q}_{\infty}=\mathbb{Q}_{2^{m}}\left(g_{i, d_{i}}^{1 / 2^{d_{i}+1}}: 0 \leqslant d_{i} \leqslant n-1\right)
$$

for all positive integers $m \geqslant n$. Writing $g_{i, d_{i}}=b_{i}^{2^{d_{i}}}$, where the $b_{i}$ 's are strongly 2-independent positive rational numbers, we then have

$$
\mathbb{Q}_{2^{m}, 2^{n}} \cap \mathbb{Q}_{\infty}=\mathbb{Q}_{2^{m}}\left(\sqrt{b_{i}}: 0 \leqslant d_{i} \leqslant n-1\right) .
$$

Proof. Notice that (5.2) is an immediate consequence of (5.1). The inclusion $\supseteq$ in (5.1) holds because the elements generating the field on the right-hand side lie in $\sqrt[2 n]{G}$, and they can be expressed as the square root of some rational number times a root of unity of order dividing $2^{n}$.

Now we prove the inclusion $\subseteq$ in (5.1). The left-hand side of (5.1) is a finite abelian extension of $\mathbb{Q}_{2^{m}}$ of exponent dividing $2^{n}$ and hence by classical Kummer theory it is of the form $\mathbb{Q}_{2^{m}}\left(H^{1 / 2^{n}}\right)$, where $H$ is a subgroup of $\mathbb{Q}_{2^{m}}^{\times}$such that $H \mathbb{Q}_{2^{m}}^{\times 2^{n}} \subseteq G \mathbb{Q}_{2^{m}}^{\times 2^{n}}$. Therefore it is sufficient to determine which $2^{n}$-th roots of elements of $G$ lie in $\mathbb{Q}_{\infty}$. 
The $2^{n}$-th root of a generator $g_{i, d_{i}}$ with $d_{i} \geqslant n-1$ lies in the field on the right-hand side, so we may suppose that $n \geqslant 2$ and reduce to study the elements of the form

$$
g=\prod_{i \in J_{0}} g_{i, 0}^{f_{i}} \prod_{i \in J_{1}} g_{i, 1}^{f_{i}} \cdots \prod_{i \in J_{n-2}} g_{i, n-2}^{f_{i}},
$$

where $J_{d}$ consists of the indices $i$ such that the generator $g_{i, d_{i}}$ has divisibility parameter $d_{i}=$ $d$. Analogously, we may restrict to consider exponents $f_{i}$ that are positive integers such that $v_{2}\left(f_{i}\right)+d_{i}<n-1$. We are left to show that no such element has a $2^{n}$-th root which lies in a cyclotomic field. We may rewrite

$$
g=\prod_{i} b_{i}^{e_{i}}
$$

such that $v_{2}\left(e_{i}\right)<n-1$, and we conclude by the following Lemma.

Lemma 5.2. Let $b_{1}, \ldots, b_{r}$ be strongly 2 -independent elements of $\mathbb{Q}^{\times}$. If $n \geqslant 2$, then no $2^{n}$-th root of a product of the form

$$
g=\prod_{i} b_{i}^{e_{i}} \quad \text { with } v_{2}\left(e_{i}\right)<n-1 \forall i
$$

belongs to a cyclotomic field.

Proof. For $n=2$ we know that the product $g$, having odd exponents, is strongly 2-indivisible in $\mathbb{Q}^{\times}$and hence its fourth root does not lie in a cyclotomic field (which is an abelian extension of $\mathbb{Q}$ ) by [6. Theorem 2] (see also [4, Theorem 3.3]). Now suppose that $n \geqslant 3$. If $v_{2}\left(e_{i}\right)=0$ for some index $i$ we may reason as above, so suppose that all exponents $e_{i}$ are even and write $\sqrt{g}=\prod_{i} b_{i}^{e_{i} / 2}$ where $v_{2}\left(e_{i} / 2\right)<n-2$. The result for $n-1$ applied to $\sqrt{g}$ gives that no $2^{n-1}$ th root of this element belongs to a cyclotomic field, so we may conclude by induction.

Theorem 5.3. Let $G$ be a finitely generated and torsion-free subgroup of $\mathbb{Q}^{\times}$of positive rank. Suppose that $G$ contains negative elements, and consider a 2-good basis $\left\{g_{i, d_{i}}\right\}$ such that exactly one of the generators, say $g_{j, x}$, is negative (cf. Remark 4.1). Then for all positive integers $m \geqslant n$ we have

$$
\mathbb{Q}_{2^{m}, 2^{n}} \cap \mathbb{Q}_{\infty}=\mathbb{Q}_{2^{v}}\left(g_{i, d_{i}}^{1 / 2^{d_{i}+1}}: 0 \leqslant d_{i} \leqslant n-1\right),
$$

where $v=m$ if $n \geqslant x+1$, and $v=\max (m, n+1)$ if $n \leqslant x$.

Writing $g_{i, d_{i}}= \pm b_{i}^{2^{d_{i}}}$, where the $b_{i}$ 's are strongly 2-independent positive rational numbers (and where the sign is negative only for $i=j$ ), we have

$$
\mathbb{Q}_{2^{m}, 2^{n}} \cap \mathbb{Q}_{\infty}=\mathbb{Q}_{2^{v}}\left(\sqrt{b_{i}}: 0 \leqslant d_{i} \leqslant n-1\right),
$$

if $n \neq x+1$, while for $n=x+1$ we have

$$
\mathbb{Q}_{2^{m}, 2^{n}} \cap \mathbb{Q}_{\infty}=\mathbb{Q}_{2^{m}}\left(\zeta_{2^{x+2}} \sqrt{b_{j}}, \sqrt{b_{i}}: 0 \leqslant d_{i} \leqslant n-1, i \neq j\right) .
$$

Proof. First notice that (5.4) and (5.5) are equivalent to (5.3): for (5.4) with $x \leqslant n$ this is because we take the root of $b_{j}$ rather than that of $g_{j}$, the ratio being a root of unity in $\mathbb{Q}_{2^{v}}$.

The inclusion $\supseteq$ in (5.3) is similar to Theorem 5.1 and it is clear if one considers that, if $n \leqslant x$, the $2^{n}$-th root of $g_{j, x}$ is a rational number times $\zeta_{2^{n+1}}$. 
Now we prove the inclusion $\subseteq$. As in the proof of Theorem 5.1 we are left to detect which $2^{n}$-th roots of the elements of $G$ are contained in a cyclotomic field.

If $n=x+1$, then the $2^{n}$-th root of the negative generator lies in a cyclotomic field and it equals $\zeta_{2^{x+2}} \sqrt{b_{j}}$. The presence of $g_{j}$ in the expression of an element of $G$ as a product of generators does not change whether its $2^{n}$-th root lies in a cyclotomic field. So again we may reduce to Theorem 5.1 to prove the inclusion $\subseteq$ in 5.5 .

If $n \geqslant x+2$, then the sign of an element of $G$ does not influence whether the $2^{n}$-th root lies in a cyclotomic field and we can proceed as in Theorem 5.1 .

If $n \leqslant x$, then the $2^{n}$-th root of the negative generator only contributes by a root of unity $\zeta_{2^{n+1}}$ and hence the presence of the negative generator in an expression for $g \in G$ does not matter. Again we may proceed as in Theorem 5.1 .

Remark 5.4. Consider the fields in (5.2), (5.4), and (5.5). To study the intersection on the lefthand side we may suppose w.l.o.g. (up to squares in $\mathbb{Q}^{\times}$) that the $b_{i}$ 's are positive squarefree integers. The smallest cyclotomic field containing $\sqrt{b_{i}}$ is then $\mathbb{Q}_{b_{i}}$ if $b_{i} \equiv 1 \bmod 4$, and $\mathbb{Q}_{4 b_{i}}$ otherwise. Let $M:=\operatorname{lcm}\left\{b_{i}\right\}$, where $i$ runs over the indices such that $1 \leqslant d_{i} \leqslant n-1$ and $g_{i, d_{i}}>0$.

(1) The smallest cyclotomic field containing (5.2) is $\mathbb{Q}_{2^{m}} M$ if $b_{i} \equiv 1 \bmod 4$ for all $1 \leqslant$ $d_{i} \leqslant n-1$, and $\mathbb{Q}_{\left[2^{m}, 4 M\right]}$ otherwise.

(2) If $n \leqslant x$, then the smallest cyclotomic field containing (5.4) is $\mathbb{Q}\left[2^{v}, M\right]$ if $b_{i} \equiv 1 \bmod 4$ for all $1 \leqslant d_{i} \leqslant n-1$, and $\mathbb{Q}_{\left[2^{v}, 4 M\right]}$ otherwise.

(3) If $n \geqslant x+1$, then the smallest cyclotomic field containing (5.4) is $\mathbb{Q}_{\left[2^{m}, M, b_{j}\right]}$ if $b_{i} \equiv$ $1 \bmod 4$ for all $1 \leqslant d_{i} \leqslant n-1$, and $\mathbb{Q}_{\left[2^{m}, 4 M, b_{j}\right]}$ otherwise.

(4) Now consider the field (5.5), and let $b:=\zeta_{2^{x+2}} \sqrt{b_{j}}$.

- If $x=0$, then the smallest cyclotomic field containing $b=\sqrt{-b_{j}}$ is $\mathbb{Q}_{b_{j}}$ if $-b_{j} \equiv$ $1 \bmod 4$, and $\mathbb{Q}_{4 b_{j}}$ otherwise.

- If $x \geqslant 1$ and $2 \nmid b_{j}$, then the smallest cyclotomic field containing $b$ is $\mathbb{Q}_{2^{x+2} b_{j}}$.

- If $x=1$ and $2 \mid b_{j}$, then the smallest cyclotomic field containing $b=\zeta_{8} \sqrt{b_{j}}$ is $\mathbb{Q}_{2 b_{j}}$, as $\zeta_{8} \sqrt{2}=\zeta_{4}+1$.

- If $x>1$ and $2 \mid b_{j}$, then the smallest cyclotomic field containing $b$ is $\mathbb{Q}_{2^{x+1}} b_{j}$ because $\mathbb{Q}_{8}\left(\zeta_{2^{x+2}} \sqrt{2}\right)=\mathbb{Q}_{2^{x+2}}$ (recall that $\left.\sqrt{2} \in \mathbb{Q}_{8}\right)$.

Hence in each of these cases the smallest cyclotomic field containing the field (5.5) is given by composing the above cyclotomic fields with $\mathbb{Q}_{M}$ or $\mathbb{Q}_{4 M}$ (according to the other $b_{i}$ 's) and with $\mathbb{Q}_{2^{m}}$.

\section{THE ADELIC FAILURE}

In this section we show how to compute the degrees $B(M, N)$ for $M, N$ with $N \mid M$ (see (2.1)). Set $n:=v_{2}(N)$. We begin with two important remarks whose proof is straight-forward and is left to the reader: 
Remark 6.1. Let $G$ be as in Theorem 5.1. We use the same notation, and in particular each generator of $G$ is written as $g_{i}=b_{i}^{2^{d_{i}}}$. Let $m \geqslant n \geqslant 1$ and $T \geqslant 1$. Let

$$
\begin{gathered}
\mathcal{S}:=\left\{b_{i}: 0 \leqslant d_{i} \leqslant n-1\right\}, \\
\mathcal{C}:=\left\{y \in \mathbb{Z}: y \equiv \prod_{i} b_{i}^{e_{i}} \bmod \mathbb{Q}^{\times 2}, b_{i} \in \mathcal{S}, e_{i} \in\{0,1\}, y \text { squarefree }\right\},
\end{gathered}
$$

and define $H$ as the following subgroup of $\mathbb{Q}^{\times}$:

- if $8 \mid T$, then $H=\langle y \in \mathcal{C}: y \mid T\rangle$,

- if $4 \| T$, then $H=\langle y \in \mathcal{C}: y \mid T, 2 \nmid y\rangle$,

- if $4 \nmid T$, then $H=\langle y \in \mathcal{C}: y \mid T, y \equiv 1 \bmod 4\rangle$.

That is, the generators $y$ for $H$ are exactly those $y \in \mathcal{C}$ such that $\sqrt{y} \in \mathbb{Q}_{T}$. In particular we have that $\mathbb{Q}(\sqrt{H}) \subseteq \mathbb{Q}_{T}$. We then have

$$
\mathbb{Q}_{2^{m}, 2^{n}} \cap \mathbb{Q}_{T}=\mathbb{Q}_{2^{w}}(\sqrt{H}),
$$

where $w:=\min \left(m, v_{2}(T)\right)$.

Remark 6.2. Let $G$ be as in Theorem 5.3 and keep the same notation. Let $m \geqslant n \geqslant 1$ and $T \geqslant 1$.

(1) If $n \leqslant x$, define $\mathcal{S}, \mathcal{C}$ and $H$ as in Remark 6.1 Then we have

$$
\mathbb{Q}_{2^{m}, 2^{n}} \cap \mathbb{Q}_{T}=\mathbb{Q}_{2^{w}}(\sqrt{H}),
$$

where $w:=\min \left(v, v_{2}(T)\right)$.

(2) If $n \geqslant x+2$, or if $n=x+1$ and $m \geqslant n+1$, define $\mathcal{S}, \mathcal{C}$ and $H$ as in Remark 6.1 (notice that $b_{j} \in \mathcal{S}$ ). Then we have

$$
\mathbb{Q}_{2^{m}, 2^{n}} \cap \mathbb{Q}_{T}=\mathbb{Q}_{2^{w}}(\sqrt{H}),
$$

where $w:=\min \left(m, v_{2}(T)\right)$.

(3) If $m=n=x+1$, consider (5.5).

- If $n=1$, then $\sqrt{-b_{j}}$ can be treated as the other $b_{i}$ 's. More precisely, we let $\mathcal{S}$ be as in Remark 6.1 but we replace $b_{j}$ by $-b_{j}$. Define $\mathcal{C}$ and $H$ as before. Then we have

$$
\mathbb{Q}_{2^{m}, 2^{n}} \cap \mathbb{Q}_{T}=\mathbb{Q}(\sqrt{H}) \text {. }
$$

- If $n>2$ and $2^{n+1} \nmid T$, or if $n=2$ and $4 \nmid T$, define

$$
\mathcal{S}:=\left\{b_{i}: g_{i}>0,0 \leqslant d_{i} \leqslant n-1\right\},
$$

and $\mathcal{C}$ and $H$ analogously to the previous remark. Then we have

$$
\mathbb{Q}_{2^{m}, 2^{n}} \cap \mathbb{Q}_{T}=\mathbb{Q}_{2^{w}}(\sqrt{H}),
$$

where $w:=v_{2}(T)$.

- If $n=2$ and $4 \| T$, define $\mathcal{S}$ as in 6.1, and $\mathcal{C}$ and $H$ analogously to Remark 6.1 Set

$$
\begin{gathered}
\mathcal{C}^{\prime}:=\left\{z \in \mathbb{Z}: z \equiv b_{j} y \bmod \mathbb{Q}^{\times 2}, y \in \mathcal{C}, z \text { squarefree }\right\} \\
H^{\prime}:=\left\langle\zeta_{4} z: z \in \mathcal{C}^{\prime}, z \text { even }, \frac{z}{2} \mid T\right\rangle
\end{gathered}
$$


Then we have

$$
\mathbb{Q}_{2^{m}, 2^{n}} \cap \mathbb{Q}_{T}=\mathbb{Q}_{4}\left(\sqrt{\left\langle H, H^{\prime}\right\rangle}\right) .
$$

- If $n \geqslant 2$ and $2^{n+1} \mid T$, define $\mathcal{S}$ as in 6.1), $\mathcal{C}$ and $H$ as in Remark 6.1. $\mathcal{C}^{\prime}$ as in 6.2), and set

$$
H^{\prime}:=\left\langle\zeta_{2^{n}} z: z \in \mathcal{C}^{\prime}, z \mid T\right\rangle
$$

Then we have

$$
\mathbb{Q}_{2^{m}, 2^{n}} \cap \mathbb{Q}_{T}=\mathbb{Q}_{2^{n}}\left(\sqrt{\left\langle H, H^{\prime}\right\rangle}\right) .
$$

In the computation of the adelic failure $B(M, N)$ we will only need to use Remarks 6.1 and 6.2 with $n=m$ and $v_{2}(T) \geqslant n$. The case distinction simplifies as follows.

Remark 6.3. Let $M, N$ be integers with $N \mid M$, and set $n:=v_{2}(N)$. Let $G$ be as in Theorem 5.1 and keep the same notation of Remark 6.1 (here $T=M)$. Then we have

$$
B(M, N)=\left[\mathbb{Q}_{2^{n}}(\sqrt{H}): \mathbb{Q}_{2^{n}}\right] .
$$

Let $G$ be as in Theorem 5.3 and keep the same notation of Remark 6.2 case by case (here $T=M)$.

(1) If $n \leqslant x$, then $B(M, N)=\left[\mathbb{Q}_{2^{w}}(\sqrt{H}): \mathbb{Q}_{2^{n}}\right]$, where $w=\min \left(n+1, v_{2}(M)\right)$.

(2) If $n \geqslant x+2$, then $B(M, N)=\left[\mathbb{Q}_{2^{n}}(\sqrt{H}): \mathbb{Q}_{2^{n}}\right]$.

(3) If $n=x+1$, we have:

- if $n=1$, then $B(M, N)=[\mathbb{Q}(\sqrt{H}): \mathbb{Q}]$;

- if $n=v_{2}(M)>2$, then $B(M, N)=\left[\mathbb{Q}_{2^{n}}(\sqrt{H}): \mathbb{Q}_{2^{n}}\right]$;

- if $n=v_{2}(M)=2$, then $B(M, N)=\left[\mathbb{Q}_{4}\left(\sqrt{\left\langle H, H^{\prime}\right\rangle}\right): \mathbb{Q}_{4}\right]$;

- if $n \geqslant 2$ and $v_{2}(M) \geqslant n+1$, then $B(M, N)=\left[\mathbb{Q}_{2^{n}}\left(\sqrt{\left\langle H, H^{\prime}\right\rangle}\right): \mathbb{Q}_{2^{n}}\right]$.

The next proposition is used to compute the adelic failure.

Proposition 6.4. Let $H \leqslant \mathbb{Q}^{\times}$be a torsion-free and finitely generated subgroup. Assume that $H$ does not contain minus a square in $\mathbb{Q}^{\times}$. Then we have

$$
\left[\mathbb{Q}_{2^{m}}(\sqrt{H}): \mathbb{Q}_{2^{m}}\right]= \begin{cases}|\bar{H}| / 2 & \text { if } m \geqslant 3 \text { and } \exists b \in H \text { with } b \equiv \pm 2 \bmod \mathbb{Q}^{\times 2}, \\ |\bar{H}| & \text { otherwise, }\end{cases}
$$

where $\bar{H}$ is a complete set of representatives of $H \mathbb{Q}^{\times 2}$ in $\mathbb{Q}^{\times} / \mathbb{Q}^{\times 2}$.

Proof. It is clear that we may replace $H$ by any other subgroup $H^{\prime} \leqslant \mathbb{Q}^{\times}$such that $H \mathbb{Q}^{\times 2}=$ $H^{\prime} \mathbb{Q}^{\times 2}$. Then we may suppose without loss of generality that $H$ is generated by squarefree integers $g_{1}, \ldots, g_{r}$, where $|\bar{H}|=2^{r}$ (notice that by hypothesis none of the $g_{i}$ 's can be -1 ). Moreover, we can also suppose that the $g_{i}$ 's are strongly 2 -independent, so that the $d$ parameters for the 2-divisibility of $H$ are all zero over $\mathbb{Q}$.

Suppose first that $m \geqslant 2$. In this case we can work over $\mathbb{Q}_{4}$ and apply formula (4.2) from Theorem 4.2. We just need to compute the parameters for the 2-divisibility of $H$ over $\mathbb{Q}_{4}$. The 
only squarefree integers which are not strongly 2 -indivisible over $\mathbb{Q}_{4}$ are \pm 2 and in particular we have

$$
2=\zeta_{4}^{-1}\left(1+\zeta_{4}\right)^{2} .
$$

A simple computation shows that if $H$ contains an element of the form \pm 2 times a square, then we may change the basis to get $g_{1}= \pm 2$.

Therefore, if $H$ does not contain \pm 2 times a square, we have that $d_{i}=0$ and $h_{i} \in\{0,1\}$ for all $i$ also over $\mathbb{Q}_{4}$, so that the formula (4.2) yields $\left[\mathbb{Q}_{2^{m}}(\sqrt{H}): \mathbb{Q}_{2^{m}}\right]=2^{r}$. Otherwise, only the parameters corresponding to $g_{1}= \pm 2$ change from $\mathbb{Q}$ to $\mathbb{Q}_{4}$ (in view of 6.3 we get the new parameters $d_{1}=1$ and $h_{1}=2$ ). Hence by (4.2) this degree will be $2^{r}$ for $m=2$ and $2^{r-1}$ for $m \geqslant 3$.

Now let $m=1$. Since $H$ does not contain minus a square, by (4.3) we get

$$
[\mathbb{Q}(\sqrt{H}): \mathbb{Q}]=\left[\mathbb{Q}_{4}(\sqrt{H}): \mathbb{Q}_{4}\right]
$$

which is $2^{r}$ by our previous computation.

Corollary 6.5. Let $G$ be a finitely generated and torsion-free subgroup of $\mathbb{Q}^{\times}$of rank $r$. Then the total adelic failure $\beta$ for $G$ is equal to

$$
\beta= \begin{cases}2^{r-1} & \text { if there is } g \in G \text { of the form } g= \pm\left(2 a^{2}\right)^{2^{d}} \\ 2^{r} & \text { otherwise }\end{cases}
$$

where $a \in \mathbb{Q}^{\times}$and where $d$ is a non-negative integer.

Proof. We know that the adelic failure can only be a power of 2 . By Remarks 6.1 and 6.2 , for $n \geqslant 2$ large enough, we have

$$
\mathbb{Q}_{2^{n}, 2^{n}} \cap \mathbb{Q}_{\infty}=\mathbb{Q}_{2^{n}}(\sqrt{H}),
$$

where $H:=\left\langle b_{1}, \ldots, b_{r}\right\rangle$, and the $b_{i}$ 's are the strongly 2 -indivisible parts of the elements of the 2 -good basis of $G$ chosen in Theorems 5.1 and 5.3. In particular the $b_{i}$ 's are strongly 2-independent over $\mathbb{Q}$ and form a 2-good basis of $H$. Therefore, $|\bar{H}|=2^{r}$, where $\bar{H}$ is a complete set of representatives of $H \mathbb{Q}^{\times 2}$ modulo $\mathbb{Q}^{\times 2}$. Hence by Proposition 6.4 the degree of $\mathbb{Q}_{2^{n}}(\sqrt{H})$ over $\mathbb{Q}_{2^{n}}$ is $2^{r-1}$ if $H$ contains an element of the form 2 times a square, and $2^{r}$ otherwise.

By [5, Proposition 5] (where $f=2$ in our setup) $H$ contains an element of the form 2 times a square if and only if there is an element in $G$ which is equal to $\pm\left(2 a^{2}\right)^{2^{d}}$.

\section{THE KUMMER FAILURE}

In this section we prove Theorem 1.1 which states that, for a fixed group $G$, there are integers $M_{0}, N_{0}$ such that the Kummer failure $C(M, N)$ only depends on $\operatorname{gcd}\left(M, M_{0}\right)$ and $\operatorname{gcd}\left(N, N_{0}\right)$. 
Proposition 7.1. Fix a prime number $\ell$. There is some integer $c_{\ell}$ such that for all $N \geqslant 1$ the $\ell$-adic failure $A_{\ell}(N)$ depends on $N$ only through $\operatorname{gcd}\left(N, \ell^{c_{\ell}}\right)$. More precisely, we have

$$
A_{\ell}(N)=A_{\ell}\left(\operatorname{gcd}\left(N, \ell^{c_{\ell}}\right)\right) .
$$

If $\ell$ is odd, then we may take for $c_{\ell}$ the maximum of the d-parameters for the $\ell$-divisibility of $G$. If $\ell=2$, then we may take $c_{2}=\max _{i}\left(d_{i}+h_{i}\right)$, where the $d_{i}$ 's and the $h_{i}$ 's are the parameters for the 2-divisibility of $G$ over $\mathbb{Q}_{4}$.

Proof. Set $n=v_{\ell}(N)$. By definition $A_{\ell}(N)$ depends only on $n$ and it is 1 if $n=0$. We only prove that, if $c_{\ell}$ is defined as in the statement, $A_{\ell}(N)$ is constant on $\left\{N: n \geqslant c_{\ell}\right\}$, because it is easy to check from our computations that in this case $A_{\ell}(N)=A_{\ell}\left(\operatorname{gcd}\left(\ell^{c_{\ell}}, N\right)\right)$.

By Proposition 3.1, for $\ell$ odd we have

$$
v_{\ell}\left(\left[\mathbb{Q}_{\ell^{n}, \ell^{n}}: \mathbb{Q}_{\ell^{n}}\right]\right)=r n-\sum_{i=1}^{r} \min \left(d_{i}, n\right),
$$

where the $d_{i}$ 's are the $d$-parameters for the $\ell$-divisibility of $G$. Therefore, if $n \geqslant c_{\ell}$ we obtain

$$
v_{\ell}\left(A_{\ell}(N)\right)=\sum_{i=1}^{r} d_{i}
$$

For $\ell=2$ and $n \geqslant 2$, by formula 4.2 from Theorem 4.2 , we have

$$
v_{2}\left(A_{2}(N)\right)=n-\max \left\{h_{i}+\min \left(n, d_{i}\right): 1 \leqslant i \leqslant r\right\} \cup\{n\}+\sum_{i=1}^{r} \min \left(d_{i}, n\right) .
$$

If $n \geqslant c_{2}$, then we clearly have

$$
v_{2}\left(A_{2}(N)\right)=\sum_{i=1}^{r} d_{i}
$$

Now let $\ell=2$ and $n=1$. If $c_{2} \geqslant 1$ the formula $A_{2}(N)=A_{2}\left(\operatorname{gcd}\left(2^{c_{2}}, N\right)\right)$ is obvious. If $c_{2}=0$ we have to prove $A_{2}(2)=A_{2}(1)$, which means $A_{2}(2)=1$.

By Theorem 4.2 , Eq. 4.3 we have $[\mathbb{Q}(\sqrt{G}): \mathbb{Q}]=\left[\mathbb{Q}_{4}(\sqrt{G}): \mathbb{Q}_{4}\right]$ if $G$ does not contain minus a square in $\mathbb{Q}^{\times}$. This is the case because the generators of $G$ are strongly 2 -independent $\left(c_{2}=0\right.$ implies that $d_{i}=0$ for all $i$ ). By Proposition 6.4 (where $m=2$ ) we have $\left[\mathbb{Q}_{4}(\sqrt{G})\right.$ : $\left.\mathbb{Q}_{4}\right]=2^{r}$. We conclude that $A_{2}(2)=1$.

Proposition 7.2. There are integers $M_{0}, N_{0}$, with $N_{0} \mid M_{0}$, such that for all integers $M, N \geqslant 1$ with $N \mid M$ the adelic failure $B(M, N)$ depends on $M$ and $N$ only through $\operatorname{gcd}\left(M, M_{0}\right)$ and $\operatorname{gcd}\left(N, N_{0}\right)$. More precisely, we have

$$
B(M, N)=B\left(\operatorname{gcd}\left(M, M_{0}\right), \operatorname{gcd}\left(N, N_{0}\right)\right) .
$$

In particular, keeping the notation of Sections 4 and 5 , we may take: 
- $N_{0}=2^{n_{0}}$, where

$$
n_{0}=\max \left\{3, x+2, d_{1}+1, \ldots, d_{r}+1\right\}
$$

where $x$ is the d-parameter for the 2-divisibility of the only negative basis element, or -1 if there is no such element.

- $M_{0}=\operatorname{lcm}\left\{N_{0}, b_{1}^{\prime}, \ldots, b_{r}^{\prime}\right\}$, where the $b_{i}^{\prime}$ 's are positive squarefree integers such that $b_{i} \equiv b_{i}^{\prime} \bmod \mathbb{Q}^{\times 2}$ (recall that the $b_{i}$ 's are positive rational numbers).

Proof. Let $M_{0}$ and $N_{0}$ be as above and set $n=v_{2}(N)$. Suppose first that $G$ contains no negative elements: we are in the setup of Theorem 5.1 and Remark 6.1. Since $G \subseteq \mathbb{Q}_{+}^{\times}$, we need to compare

$$
\begin{gathered}
B(M, N)=\left[\mathbb{Q}_{2^{n}}(\sqrt{H}): \mathbb{Q}_{2^{n}}\right] \quad \text { and } \\
B\left(\operatorname{gcd}\left(M, M_{0}\right), \operatorname{gcd}\left(N, N_{0}\right)\right)=\left[\mathbb{Q}_{2^{e}}(\sqrt{H}): \mathbb{Q}_{2^{e}}\right],
\end{gathered}
$$

where $e:=\min \left(n, n_{0}\right)$. Notice that the groups $H$ are the same because we have the same sets $\mathcal{S}$ and $\mathcal{C}$ for both failures, and for every $y \in \mathcal{C}$ we have $\sqrt{y} \in \mathbb{Q}_{M}$ if and only if $\sqrt{y} \in \mathbb{Q}_{\left(M, M_{0}\right)}$. By Proposition 6.4 we easily deduce that $\left[\mathbb{Q}_{2^{n}}(\sqrt{H}): \mathbb{Q}_{2^{n}}\right]=\left[\mathbb{Q}_{2^{e}}(\sqrt{H}): \mathbb{Q}_{2^{e}}\right]$.

Now suppose that $G$ contains negative elements: we are in the setup of Theorem 5.3 and Remark 6.2. For $B\left(\left(M, M_{0}\right),\left(N, N_{0}\right)\right)$ we find the same case distinction of Remark 6.3 for $B(M, N)$. Again, the sets $\mathcal{S}, \mathcal{C}$, and $H$ (as well as $\mathcal{C}^{\prime}$ and $H^{\prime}$ ) are the same in both cases. Notice that $v_{2}\left(M_{0}\right)=n_{0}$.

- If $n \leqslant x$, then we have

$$
\begin{gathered}
B(M, N)=\left[\mathbb{Q}_{2^{u}}(\sqrt{H}): \mathbb{Q}_{2^{n}}\right] \quad \text { and } \\
B\left(\operatorname{gcd}\left(M, M_{0}\right), \operatorname{gcd}\left(N, N_{0}\right)\right)=\left[\mathbb{Q}_{2^{w}}(\sqrt{H}): \mathbb{Q}_{2^{n}}\right],
\end{gathered}
$$

where $u:=\min \left(n+1, v_{2}(M)\right)$ and $w:=\min \left(v_{2}\left(\left(N, N_{0}\right)\right)+1, v_{2}\left(\left(M, M_{0}\right)\right)\right)$. Since $\left(N, N_{0}\right)=2^{n}$ and $n_{0} \geqslant x+2 \geqslant n$, we easily see that $w=u$, so that $B\left(\left(M, M_{0}\right),\left(N, N_{0}\right)\right)=B(M, N)$.

- If $n \geqslant x+2$, then $B\left(\left(M, M_{0}\right),\left(N, N_{0}\right)\right)=\left[\mathbb{Q}_{2^{e}}(\sqrt{H}): \mathbb{Q}_{2^{e}}\right]$, where $e=\min \left(n, n_{0}\right)$, so that we can argue as in the case $G \subseteq \mathbb{Q}_{+}^{\times}$.

- If $n=x+1$, for all subcases the equality of the two failures follows directly because $v_{2}\left(\left(N, N_{0}\right)\right)=n$ as $n \leqslant n_{0}$.

Corollary 7.3. Let $\ell$ be a prime number.

(1) There is an integer $c_{\ell}$ (depending only on $G$ and $\ell$ ) such that for all $N \geqslant 1$ with $v_{\ell}(N) \geqslant c_{\ell}$ we have $A_{\ell}(N)=A_{\ell}\left(\ell^{c_{\ell}}\right)$. We may take $c_{\ell}$ as in Proposition 7.1. Then the total $\ell$-adic failure is given by $\alpha_{\ell}=A_{\ell}\left(\ell^{c_{\ell}}\right)$.

(2) There are integers $M_{0}, N_{0}$ with $N_{0} \mid M_{0}$ (depending only on $G$ ) such that for all $N, M$ with $N\left|M, M_{0}\right| M$ and $N_{0} \mid N$ we have $B(M, N)=B\left(M_{0}, N_{0}\right)$. We may take $M_{0}$, $N_{0}$ as in Proposition 7.2 Then the total adelic failure is given by $\beta=B\left(M_{0}, N_{0}\right)$. 
Remark 7.4. With the notation of Proposition 7.1 if $N_{\ell}$ is a multiple of $\ell^{c_{\ell}}$, then we have

$$
A_{\ell}(N)=A_{\ell}\left(\operatorname{gcd}\left(N, N_{\ell}\right)\right) \quad \text { for all } N \geqslant 1 \text {. }
$$

With the notation of Proposition 7.2 if $M_{0}^{\prime}$ and $N_{0}^{\prime}$ are multiples of $M_{0}$ and $N_{0}$, respectively, and satisfy $N_{0}^{\prime} \mid M_{0}^{\prime}$, then we have

$$
B(M, N)=B\left(\operatorname{gcd}\left(M, M_{0}^{\prime}\right), \operatorname{gcd}\left(N, N_{0}^{\prime}\right)\right) \quad \text { for all } M, N \geqslant 1 \text { with } N \mid M .
$$

Theorem 7.5. There are integers $M_{0}$ and $N_{0}$ such that, for all integers $N, M$ with $N \mid M$, the Kummer failure $C(M, N)$ depends on $M$ and $N$ only through $\operatorname{gcd}\left(M, M_{0}\right)$ and $\operatorname{gcd}\left(N, N_{0}\right)$. More precisely, we have

$$
C(M, N)=C\left(\operatorname{gcd}\left(M, M_{0}\right), \operatorname{gcd}\left(N, N_{0}\right)\right) .
$$

In particular, keeping the notation of the previous sections, we may take:

- $N_{0}=\prod_{\ell} \ell^{c_{\ell}}$, where $c_{\ell}=\max _{i} d_{i}$ if $\ell \neq 2$, the $d_{i}$ 's being the $d$-parameters for the $\ell$-divisibility of $G$ (notice that $c_{\ell}=0$ for all but finitely many primes $\ell$ ), and

$$
c_{2}=\max \left\{3, d_{1}+h_{1}+1, \ldots, d_{r}+h_{r}+1\right\},
$$

where $d_{i}$ and $h_{i}$ are the parameters for the 2-divisibility of $G$ over $\mathbb{Q}_{4}$.

- $M_{0}=\operatorname{lcm}\left\{N_{0}, b_{1}^{\prime}, \ldots, b_{r}^{\prime}\right\}$, where the $b_{i}^{\prime}$ 's are positive squarefree integers such that $b_{i} \equiv b_{i}^{\prime} \bmod \mathbb{Q}^{\times 2}$.

In particular, the total Kummer failure is given by $C_{0}=C\left(M_{0}, N_{0}\right)$.

Proof. By (2.2) we have $C(M, N)=B(M, N) \prod_{\ell} A_{\ell}(N)$. Therefore it suffices to combine Propositions 7.1 and 7.2 and to take into account the previous remark.

\section{EXAMPLES}

In this last section we work out a few examples to illustrate the procedure described in the rest of the article.

Example 8.1. Let $G=\left\langle 5^{4}, 7\right\rangle$. We compute the $\ell$-adic failure for all primes $\ell$ and the adelic failure. For $\ell$ odd, the basis $\left\{5^{4}, 7\right\}$ is an $\ell$-good basis consisting of strongly $\ell$-independent elements. Therefore if $\ell \neq 2$ we have $A_{\ell}(N)=1$ for all $N \geqslant 1$. For $\ell=2$, the basis $\left\{5^{4}, 7\right\}$ is 2-good over $\mathbb{Q}\left(\zeta_{4}\right)$ as 5,7 are strongly 2 -independent. Therefore $G$ has parameters $d_{1}=2$, $d_{2}=h_{1}=h_{2}=0$, which by Theorem 4.2 gives

$$
\left[\mathbb{Q}_{2^{n}}\left(G^{1 / 2^{n}}\right): \mathbb{Q}_{2^{n}}\right]=2^{2 n-2} \quad \text { for all } n \geqslant 2,
$$

and $[\mathbb{Q}(\sqrt{G}): \mathbb{Q}]=2$. Hence the 2 -adic failure is given as follows, where $n=v_{2}(N)$ :

$$
A_{2}(N)= \begin{cases}1, & \text { if } n=0 \\ 2, & \text { if } n=1 \\ 4, & \text { if } n \geqslant 2 .\end{cases}
$$

Next we compute the adelic failure. Let $M, N \geqslant 1$ be integers with $N \mid M$. We apply the method outlined in Remark 6.1, and Proposition 6.4 It is trivial that $B(M, N)=1$ if $n=0$. 
Suppose that $n=1$ or $n=2$, so that $\mathcal{S}=\{7\}$. Then we have $H=\langle 7\rangle$ and so $B(M, N)=2$ if $28 \mid M$, and $H=\langle 1\rangle$ so that $B(M, N)=1$ otherwise. Suppose that $n \geqslant 3$, so that $8 \mid M$ and $\mathcal{S}=\{5,7\}$. Then we have

- $H=\langle 1\rangle$ and $B(M, N)=1$ if $5 \nmid M$ and $7 \nmid M$,

- $H=\langle 5\rangle$ and $B(M, N)=2$ if $5 \mid M$ and $7 \nmid M$,

- $H=\langle 7\rangle$ and $B(M, N)=2$ if $5 \nmid M$ and $7 \mid M$,

- $H=\langle 5,7\rangle$ and $B(M, N)=4$ if $35 \mid M$.

Example 8.2. Let us compute the adelic failure for the group $G=\langle-5,7\rangle$. Let $M, N \geqslant 1$ be such that $N \mid M$ and $v_{2}(N) \geqslant 1$. Since the $d$-parameters for the 2-divisibility are both zero, if $v_{2}(N)=1$ we have $\mathcal{S}=\{-5,7\}$, so that

- $H=\langle 1\rangle$ and $B(M, N)=1$ if $20 \nmid M, 28 \nmid M$, and $35 \nmid M$,

- $H=\langle-5\rangle$ and $B(M, N)=2$ if $20 \mid M$ and $28 \nmid M$,

- $H=\langle 7\rangle$ and $B(M, N)=2$ if $20 \nmid M$ and $28 \mid M$,

- $H=\langle-35\rangle$ and $B(M, N)=2$ if $35 \mid M$ and $4 \nmid M$,

- $H=\langle-5,7\rangle$ and $B(M, N)=4$ if $140 \mid M$.

On the other hand, if $v_{2}(N) \geqslant 2$, then the fourth root of -5 does not lie in $\mathbb{Q}_{\infty}$ but $\sqrt{5}$ lies in $\mathbb{Q}\left(\zeta_{5}\right)$. Hence $\mathcal{S}=\{5,7\}$ and since $4 \mid M$ we obtain exactly the same cases as in the previous example for $v_{2}(N) \geqslant 3$.

Example 8.3. Let us compute the adelic failure for the group $G=\left\langle 5,-7^{8}\right\rangle$. Let $M, N \geqslant 1$ be such that $N \mid M$ and $n:=v_{2}(N) \geqslant 1$. The $d$-parameters for the 2-divisibility are $d_{1}=0$ and $d_{2}=3$. If $n \leqslant 3$ then $\mathcal{S}=\{5\}$ and the $2^{n}$-th root of $-7^{8}$ yields a root of unity of order $2^{n+1}$. Thus we have

$$
\mathbb{Q}_{2^{n}, 2^{n}} \cap \mathbb{Q}_{M}=\mathbb{Q}_{2^{w}}(\sqrt{H})
$$

where $w=\min \left(n+1, v_{2}(M)\right)$ and $H=\langle 1\rangle$ if $5 \nmid M, H=\langle 5\rangle$ otherwise. Hence

- $B(M, N)=1$ if $5 \nmid M$ and $v_{2}(M)=n$,

- $B(M, N)=2$ if $5 \mid M$ and $v_{2}(M)=n$,

- $B(M, N)=2$ if $5 \nmid M$ and $v_{2}(M) \geqslant n+1$,

- $B(M, N)=4$ if $5 \mid M$ and $v_{2}(M) \geqslant n+1$.

If $n=4$, then $\mathcal{S}=\{5\}$ and the 16 -th root of $-7^{8}$ yields $\zeta_{32} \sqrt{7}$. Therefore $v_{2}(M)=4$ gives

- $H=\langle 1\rangle$ and $B(M, N)=1$ if $5 \nmid M$,

- $H=\langle 5\rangle$ and $B(M, N)=2$ if $5 \mid M$.

If $v_{2}(M) \geqslant 5$, then we obtain

- $\left\langle H, H^{\prime}\right\rangle=\langle 1\rangle$ and $B(M, N)=1$ if $5 \nmid M$ and $7 \nmid M$,

- $\left\langle H, H^{\prime}\right\rangle=\langle 5\rangle$ and $B(M, N)=2$ if $5 \mid M$ and $7 \nmid M$,

- $\left\langle H, H^{\prime}\right\rangle=\left\langle\zeta_{16} 7\right\rangle$ and $B(M, N)=2$ if $5 \nmid M$ and $7 \mid M$,

- $\left\langle H, H^{\prime}\right\rangle=\left\langle 5, \zeta_{16} 7\right\rangle$ and $B(M, N)=4$ if $35 \mid M$.

If $n \geqslant 5$ we obtain again the same cases as in Example 8.1 for $n \geqslant 3$. 
Example 8.4. We compute the 2 -adic and the adelic failure for the group $G=\left\langle 2,-7^{2}\right\rangle$. Let $N \geqslant 2$ with $n:=v_{2}(N) \geqslant 1$. The parameters for the 2-divisibility of $G$ over $\mathbb{Q}\left(\zeta_{4}\right)$ are $d_{1}=1, h_{1}=2, d_{2}=h_{2}=1$. Then applying formula (4.2) to compute the 2-adic Kummer degrees over $\mathbb{Q}\left(\zeta_{4}\right)$, we find that $A_{2}(N)=1$ if $n=1, A_{2}(N)=2$ if $n=2$, and $A_{2}(N)=4$ if $n \geqslant 3$.

Let $M \geqslant 1$ be such that $N \mid M$. The $d$-parameters for the 2-divisibility of $G$ over $\mathbb{Q}$ are $d_{1}=0$ and $d_{2}=1$. Then for $n=1$ we have $\mathcal{S}=\{2\}$ and, taking into account that the square root of $-7^{2}$ yields $\zeta_{4}$, we obtain that $B(M, N)=1$ if $2 \| M, B(M, N)=2$ if $4 \| M$, and $B(M, N)=4$ if $8 \mid M$.

If $n=2$ and $4 \| M$, then we need to take into account that $\zeta_{8} \sqrt{14}$ lies in $\mathbb{Q}\left(\zeta_{28}\right)$. We have $\mathcal{S}=\{2\}, H=\langle 1\rangle$, and we obtain

- $\left\langle H, H^{\prime}\right\rangle=\langle 1\rangle$ and $B(M, N)=1$ if $7 \nmid M$,

- $\left\langle H, H^{\prime}\right\rangle=\left\langle\zeta_{4} \cdot 14\right\rangle$ and $B(M, N)=2$ if $7 \mid M$.

If $n=2$ and $8 \mid M$, then $\mathcal{S}=\{2\}, H=\langle 2\rangle$, and we have

- $\left\langle H, H^{\prime}\right\rangle=\langle 2\rangle$ and $B(M, N)=2$ if $7 \nmid M$,

- $\left\langle H, H^{\prime}\right\rangle=\left\langle 2, \zeta_{4} \cdot 7\right\rangle$ and $B(M, N)=4$ if $7 \mid M$.

If $n \geqslant 3$, then $\mathcal{S}=\{2,7\}$ and, since $\sqrt{2} \in \mathbb{Q}\left(\zeta_{8}\right)$, we have

- $H=\langle 2\rangle$ and $B(M, N)=1$ if $7 \nmid M$,

- $H=\langle 2,7\rangle$ and $B(M, N)=2$ if $7 \mid M$.

All these examples have been tested with the SageMath implementation [7].

\section{REFERENCES}

[1] Debry, C. - PeruccA, A., Reductions of algebraic integers, J. Number Theory, 167 (2016), 259-283.

[2] Palenstijn, W. J., Radicals in arithmetic, PhD thesis, University of Leiden (2014), available at https: //openaccess.leidenuniv.nl/handle/1887/25833

[3] PerucCA, A., The order of the reductions of an algebraic integer, J. Number Theory, 148 (2015), 121-136.

[4] Perucca, A. - SgobBa, P., Kummer theory for number fields and the reductions of algebraic numbers, Int. J. Number Theory, 15 (2019), no. 08, pp. 1617-1633.

[5] Perucca, A. - Sgobba, P. - Tronto, S., Addendum to: Reductions of algebraic integers [J. Number Theory 167 (2016) 259-283], J. Number Theory, 209 (2020), 391-395.

[6] Schinzel, A., Abelian binomials, power residues and exponential congruences, Acta Arith. 32(3) (1977) 245-274. Addendum 36 (1980) 101-104; Andrzej Schinzel Selecta, Vol. 2 (European Mathematical Society, 2007), pp. 939-970.

[7] Tronto, S., Kummer Degrees, SageMath implementation and documentation available at https:// github.com/sebastianotronto/kummer-degrees

Department of Mathematics, University of Luxembourg, 6 AV. De LA Fonte, 4364 Esch-SurAlzetTe, LuXEMBOURG

Email address: antonella.perucca@uni.lu, pietro.sgobba@uni.lu, sebastiano.tronto@uni.lu 